\title{
La heterogeneización de magnitudes en la teoría tridimensional de la contabilidad*
}

\author{
The heterogeneity of magnitudes in the three-dimensional \\ accounting theory
}

\section{A heterogeneidade das magnitudes na teoria da contabilidade tridimensional}

\begin{abstract}
Carlos Alberto García-Montaño
Magíster en Gestión Empresarial de la Universidad Libre de Cali. Contador público de la Universidad de la Amazonia, con especializaciones en Administración financiera de la Universidad de la Gran Colombia-Armenia y Pedagogía y Docencia Universitaria de la Universidad de la Gran Colombia-Bogotá. Miembro de RED ICEAC y miembro de la Comisión de Administración y Finanzas de AIC. Director del Programa de Contaduría Pública de la Universidad Pedagógica y Tecnológica de Colombia, Facultad Seccional Chiquinquirá, Colombia.

alacarga8@yahoo.es
\end{abstract}

\section{Dora Marcela Rodríguez-García}

MBA - Dirección y Administración del Instituto Superior de Educación, Administración y Desarrollo ISEAD Escuela de Negocios asociada al Centro Universitario Villanueva, adscrito a la Universidad Complutense de Madrid, España. Magíster Dirección y Administración de Empresas UPTC, España. Contadora Pública Universidad Pedagógica y Tecnológica de Colombia UPTC, Especialista en Control de Gestión y Revisoría Fiscal Universidad la Gran Colombia, Docente de tiempo completo del Programa de Contaduría Pública de la Universidad Pedagógica y Tecnológica de Colombia, Facultad Seccional Chiquinquirá, Colombia domarcelar@hotmali.com

\section{Juan Carlos Ruiz-Torres}

Magíster en Administración Económica y Financiera de la Universidad Tecnológica de Pereira, UTP. Contador Público de la UPTC, Especialista en finanzas UPTC. Docente de tiempo completo del Programa de Contaduría Pública de la Universidad Pedagógica y Tecnológica de Colombia, Facultad Seccional Chiquinquirá, Colombia.

jcrtcontador@gmail.com

\section{Resumen}

Sin duda alguna la contabilidad y la profesión contable están atravesando por momentos de transformación, nuevas regulaciones, nuevos planteamientos, exposición de nuevas estructuras; la contabilidad vista desde otros ángulos, quizás en el pasado ignorados, pero hoy con la imperante necesidad de cuidar el medio ambiente como único recurso al que se debe respetar y no malgastar, hacen emergencia teorías como la Teoría Tridimensional

* Cómo citar: García-Montaño, C. A., Rodríguez-García, D. M., Ruiz-Torres. J.C. (2016). La heterogeneización de magnitudes en la teoría tridimensional de la contabilidad. Revista Libre Empresa. 13(1), 161-176 http://dx.doi.org/10.18041/libemp.2016.v13n1.25107 
de la Contabilidad, (en adelante T3C) que a través de un planteamiento serio permiten la oportunidad de presentar un aporte en lo atinente a la posibilidad de tener unidades de medida heterogéneas en contabilidad; esa es la pretensión del presente documento; en principio dejar sentado que la medición dista mucho de ser valoración económica (concepto erróneo con el que muchos contables han vivido) y que en la contabilidad se utilizan varias escalas para medir, y también metodologías y criterios para realizar valoración; es una segunda intención presentar una serie de magnitudes y de unidades de medida distintas a las monetarias, con las cuales se podría hacer representaciones contables de dimensiones distintas a la económicofinanciera, y, por último, motivar a los lectores a vincularse a estos procesos investigativos, a generar nuevas alternativas de desarrollo para una disciplina científica joven que requiere de los aportes serios de investigadores para su evolución, a arriesgarse a contrariar los pensamientos clásicos que encasillaron a la contabilidad, a los que la miraron con una visión reduccionista, representando exclusivamente la dimensión económico-financiera.

\section{Palabras clave}

Medición en contabilidad, homogeneización de magnitudes en contabilidad, heterogeneización de magnitudes en contabilidad, dimensiones contables.

\section{Abstract}

Definitely, accounting and accounting profession are going through moments of transformation, new regulations, new approaches, exposure to new structures. Accounting is seen from different points of view in time, in the past, It was unknown, but nowadays with prevalent need to care the environment as the only reserve, the one people must respect and keep, it emerges theories such as accounting three-dimensional theory (from now on T3C), that shows information related to the (possibility to have heterogeneous units of measure in accounting; that is the target for the current paper. In first instance, to establish that measurement is far to be an economic valuation (wrong concept that a lot of accounting professionals have lived with) and accounting uses some scales to measure, it also uses mythologies and criteria to assess. On second instance, to present a series of magnitudes and measurement units different from monetary ones. In that sense it would be possible to make accounting representations of different dimensions from the economic - financial one. Finally, to encourage readers to engage to these research processes, to create new development alternatives to a young scientific discipline that requires from the contributions of serious researchers to its progress. It also encourages to take the risk to speak against those classic thoughts that pigeonhole accounting, to those that have seen it with a reductionist view, by representing the economic- financial dimension only.

\section{Keywords}

Measurement in accounting, homogenization of magnitudes in accounting, heterogenization of magnitudes in accounting, accounting dimensions.

\section{Resumo}

Sem dúvida a contabilidade e a profissão de contabilidade estão passando por momentos de transformação e novas abordagens, novas regulamentações, exposição de novas estruturas; 
contabilidade vista de outros ângulos, talvez no passado ignorado, mas hoje com a necessidade vigente de cuidar do meio ambiente como remédio que deve ser respeitado e não frita fora, fazer teorias de emergência tais como a teoria tridimensional da contabilidade, (mais tarde T3C) através de uma abordagem séria permitem a oportunidade de apresentar uma contribuição para a possibilidade de ter heterogêneas contabilidade de unidades de medida; é esse o objetivo do presente documento, em princípio fazem clara essa medida está longe de ser a valoração econômica (o equívoco que muitos contabilistas viveram) várias escalas são usadas para medir na contabilidade e também metodologias e critérios de avaliação, é uma segunda intenção de apresentar uma série de quantidades e unidades de medida que não a monetária que é poderiam fazer com representações de contabilidade de diferente as dimensões económicas e financeiras e, finalmente, motivar os leitores para vincular a esses processos investigativas para gerar novas alternativas de desenvolvimento para um jovem disciplina científica que requer entrada séria de pesquisadores por sua evolução para antagonizar os pensamentos clássicos que classificado para a contabilidade, a quem olhou para ele com uma visão reducionista, que representa apenas a dimensão econômica e financeira de risco.

\section{Palavras-chave}

Medição em contabilidade, homogeneização de magnitudes em contabilidade, heterogeneización de magnitudes em dimensões de contabilidade, contabilidade.

"El hombre no puede seguir viendo la naturaleza como un prostíbulo al que solo acude para satisfacer los placeres que le brinda el modernismo instrumental”.

Oscar Raúl Sandoval Z.

"Se requiere medir con el propósito de proteger y conservar,
más que con finalidades de producción y consumo. El fin
último debe ser la sostenibilidad en términos integrales".
Mejía, Montilla y Montes

\section{Introducción}

A través de los años, la contabilidad ha sido fuente de información para los más diversos usuarios, pero el hecho de que se haya realizado siempre o casi siempre utilizando como unidad de medida alguna moneda, ha encasillado a la contabilidad en lo eminentemente monetario y que los desarrollos de la misma hayan sido o estado relacionados específicamente con esta dimensión.

Esta tendencia de representar únicamente la dimensión económico-financiera de la realidad, ha provocado un anquilosamiento de la contabilidad no financiera, sin embargo, se escucharon las voces de inconformidad de varios autores que demandaron el establecimiento de otras dimensiones en la contabilidad que dieran cuenta de situaciones que ocurren en la realidad social, en relación con el ambiente. 
Cuando se consideran otras dimensiones de la contabilidad como la social, o la ambiental, se abre un panorama distinto, más amplio para la contabilidad, otras posibilidades para que la contabilidad realice representaciones de la realidad distintas a la realidad económicafinanciera.

\section{Materiales y métodos}

El presente artículo es el resultado de los avances de una investigación de enfoque cualitativo toda vez que se ha hecho una revisión de la concepción tanto de la medición como de la valoración y se realiza una distinción clara entre lo que es la medición física y la valoración económica, términos que han sido utilizados dentro de la contabilidad en forma indiscriminada y como si fueran sinónimos.

Metodológicamente se ha realizado el establecimiento y uso de los conceptos de medición, medición física, medición cuantitativa monetaria, valoración económica o financiera, medición del valor; desde el entendido de los diferentes autores y a través del tiempo.

Con la propuesta de la T3C, en donde los autores plantean el uso de otro tipo de mediciones, se hace necesario precisar estos términos, se debe tener absoluta claridad de los conceptos ya que se está en la búsqueda del establecimiento de unidades de medida cuantitativas no monetarias, que no sea necesario homogeneizar y unidades de medida cualitativas que a la vez permitan el desarrollo de las dimensiones social y ambiental de la contabilidad.

En La Figura 1 se puede evidenciar, a través del flujograma de información, cuándo se está realizando una valoración financiera, cuándo se está haciendo una medición del valor, cuándo se establece una medición física e inclusive cuándo no se puede hacer medición.

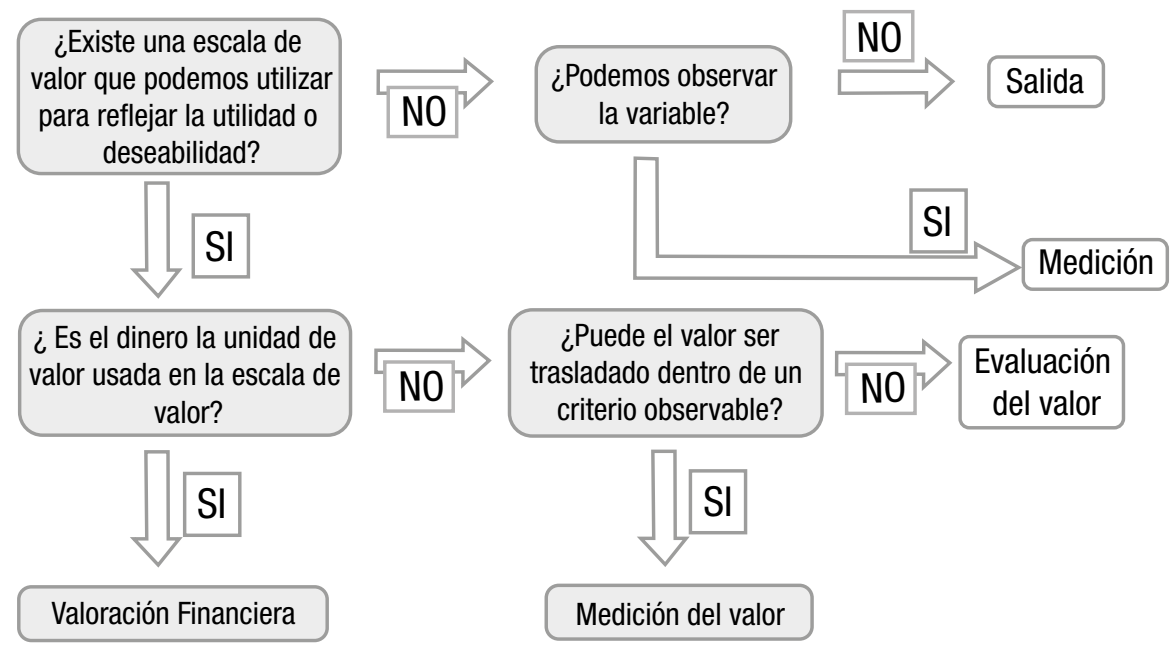

Figura 1. Medición, medición del valor, evaluación del valor y valoración financiera.

Fuente: Andriessen, 2004 pág. 230 


\section{La medición}

La medición ha sido requerida en el desarrollo de gran parte de las disciplinas, el hecho de que se mida en forma cuantitativa o cualitativa no desdibuja la intención clara de demostrar la importancia que tiene la medición para la evolución científica.

Díez Nicolás (1969, citado por Requena, 1977, p. 63) manifiesta que "lo cuantitativo no quita lo cualitativo, es decir, no hay por qué presentar esos dos campos de investigación como excluyentes".

Para Requena (1977, p. 63 y 64)) aun cuando la medición responde a funciones matemáticas, no significa exactitud sino dimensionalidad por cuanto no debe confundirse exactitud con rigor. Una ciencia histórica no es exacta, pero no por ello deja de ser rigurosa; para la ordenación de los hechos en la historia pueden aplicarse una o más escalas de medición, sin que ello quiera decir que sea exacta, aunque sí igualmente rigurosa que las ciencias formales.

La función de medición (Stevens, 1959, citado por Mattessich, 2002, p. 56) se entiende como la asignación de numerales a objetos o sucesos de acuerdo con reglas, se puede concluir que la medición implica un proceso de abstracción de alguno de los aspectos de los distintos sucesos, fenómenos o realidades a medir, que se establece, por un principio de equivalencia una relación representativa con conjuntos similares o diferentes, que suelen tener una unidad del sistema fácilmente identificable y agregable.

En ese mismo sentido y tratando de completar la definición de Stevens, Ackoff (1962, citado por Mattessich, 2002, p. 56 y 57) define a la medición como "el procedimiento por el cual obtenemos símbolos que pueden ser usados para representar el concepto a definir". Los numerales son una forma de presentación de símbolos, pero no la única.

Realizando un estudio más detallado el profesor IJIRI Y. (1967), plantea que el proceso de medición está integrado por tres factores principales:

1.- Un objeto cuya propiedad va a ser medida.

2.- Un sistema de medición integrado por un conjunto de reglas e instrumentos.

3.- Una persona que realiza la medición.

Equivocadamente los conceptos de la magnitud y de la cantidad se utilizan en forma sinonímica, aspecto que generalmente confunde tanto a los preparadores como a los usuarios de la información financiera.

En su estudio el profesor Requena (1977, p. 67), utiliza lo definido por Rey Pastor en su curso cíclico de Matemáticas en la cual expresa así: "suele definirse la magnitud como todo lo capaz de aumento o disminución”, argumentando que el propio autor agrega que este concepto carece de precisión para que pueda servir de base a ningún estudio científico. 
En esta misma línea y siguiendo al autor, medir es hacer corresponder a cada uno de los grados o estados en que aparece una misma especie de magnitud, según determinados criterios de igualdad y de orden, un número o sistema de números que puedan concebirse como una variable correspondiente a cada uno de los estados (llamados cantidades) de la magnitud en cuestión.

Conforme a algunos autores, la contabilidad no mide (Habdelgani y Lemos de la Cruz, 2010) toma elementos de la economía y de la teoría económica para cumplir con su objetivo de representar la realidad económica. En palabras de los autores "De una u otra forma el procedimiento que se adopta para registrar los precios no es medición, son relaciones, cocientes, proporciones, valores numéricos con significado monetario, que permiten comparaciones en el tiempo; configurar valores numéricos con significado monetario y sustraer interpretaciones conforme a los objetivos, intereses y teorías de los usuarios”.

\section{La contabilidad}

La contabilidad es un saber estratégico imbricado en la sociedad, cuyo objeto de estudio es la variación cuali-cuantitativa de los recursos que son controlados por las organizaciones económicas, de altísima importancia social, que ha evolucionado de manera significativa en el último cincuentenario.

Aunque la contabilidad tiene cientos de definiciones para los efectos de este trabajo investigativo, entre las concepciones antiguas, más tenidas en cuenta se encuentra la establecida por René Edgard Delaporte (1685, citado por Tua 1995, p. 146-147).

La contabilidad es la ciencia de las cuentas, que representa los movimientos de los valores de cambio clasificados en sus funciones principales y accesorias. Trata de las concepciones, coordinaciones, transformaciones, y clasificaciones de todos los valores materiales o jurídicos, servicios, desembolsos y otros que influyen en las situaciones de los patrimonios privados o públicos.

Entre las definiciones más recientes se encuentra Mejía, Mora y Montes (2013, p. 181). La contabilidad es la ciencia social aplicada que estudia la valoración cualitativa y cuantitativa de la existencia y circulación de la riqueza controlada por la organización (riqueza en su dimensión: ambiental, social y económica), utilizando diversos métodos que le permiten cumplir su función de evaluar la gestión que la organización ejerce sobre la riqueza, con el fin de contribuir a la óptima acumulación, generación, distribución y sostenibilidad integral de la mencionada riqueza.

\section{La medición en contabilidad}

Para el profesor Requena (1977, p. 66) la aplicación de la medición a la ciencia de la Contabilidad podría concretarse, en la asignación de numerales al conjunto de fenómenos económicos pasados, presentes o futuros de una unidad, de acuerdo con unas reglas, que se denominan escalas de medición, siempre que éstas cumplan las condiciones determinativa y de no degeneración, en el sentido de que sea posible la asignación de diferentes numerales a diferentes cosas o a una misma cosa bajo diferentes condiciones. 
Dentro de estas escalas consideradas en la contabilidad tenemos la escala nominal basada en principio en la clasificación, la cual fue utilizada para el establecimiento de los cuadros de cuentas, también hacen parte de estas la escala ordinal, de intervalo y proporcionales.

Siguiendo el estudio del profesor Requena existen varias clases de medición, entre ellas la directa y la indirecta que puede ser asociativa, la derivada y por confianza; entre estas clases de medición, la derivada es la que alcanza más importancia en nuestra ciencia, toda vez que la mayor parte de las magnitudes contables son de naturaleza compuesta y, por consiguiente, su medición ha de llevarse a cabo a través de los valores que tome la razón entre dos o más cantidades de otras magnitudes simples o compuestas e independientes de la que se pretende medir, pero ligadas a ésta por una ley que define la expresión funcional que describe su medición indirecta.

\section{La valoración o medición económica}

Para Rescher (1969) la valoración es una evaluación o medición comparativa de alguna cosa con respecto a su representación de un cierto valor. En la valoración se puede hacer uso de una escala de valores, siempre y cuando el valor de dicha escala sea encontrado para ser presentado en un caso particular relacionado con variaciones en grados, esta puede ser una escala ordinal que refleje la variación de los grados de valor, pero no muestra el intervalo en la posición en la escala.

Se admite por los economistas que la noción de valor en cambio o valor social de una mercancía es su poder o capacidad de compra, que consiste en el poder o capacidad que transmite a quien la posee, de obtener a trueque de ella, cualquier otro bien o servicio. De otra parte, la noción de valor se admite como poder o capacidad de compra. El precio es la razón de cambio o proporción de intercambio del dinero con la mercancía o la suma de dinero que se puede obtener mediante la cesión de una unidad de mercancía. Zamora (1989, citado por Habdelgani y Lemos de la Cruz, 2010, p. 106).

\section{La valoración en contabilidad}

A partir de los criterios establecidos para efectuar las valoraciones en contabilidad entre los cuáles se tienen el costo histórico, el valor de realización, el valor de reposición, el valor presente, el valor razonable, el neto de realización, entre otros, se puede clarificar que siempre para los cálculos de estas mediciones económicas, deberá tenerse en cuenta un elemento clave, el precio, y este a su vez es un producto resultante de los fenómenos de la oferta y la demanda que son estudiados por la economía.

La contabilidad permite tomar los precios que se asignan como resultado de la oferta y la demanda al activo y llama beneficios al resultado que se obtiene por la ejecución de los derechos o servicios expresable a través del precio; no realiza mediciones de propiedades de los objetos que se subsumen a las transacciones, toma o registra precios Hendriksen (1999, citado por Habdelgani y Lemos de la Cruz, 2010, p. 110). 
Este particular hecho de tomar sempiternamente la variable precio $u$ ocuparse exclusivamente de las situaciones económicas, ha reducido o limitado a la contabilidad a presentar única y específicamente la dimensión económico-financiera, dimensión bajo la cual se ha desarrollado la contabilidad financiera y que no ha permitido el desarrollo de la contabilidad no financiera (Ver Figura 2).

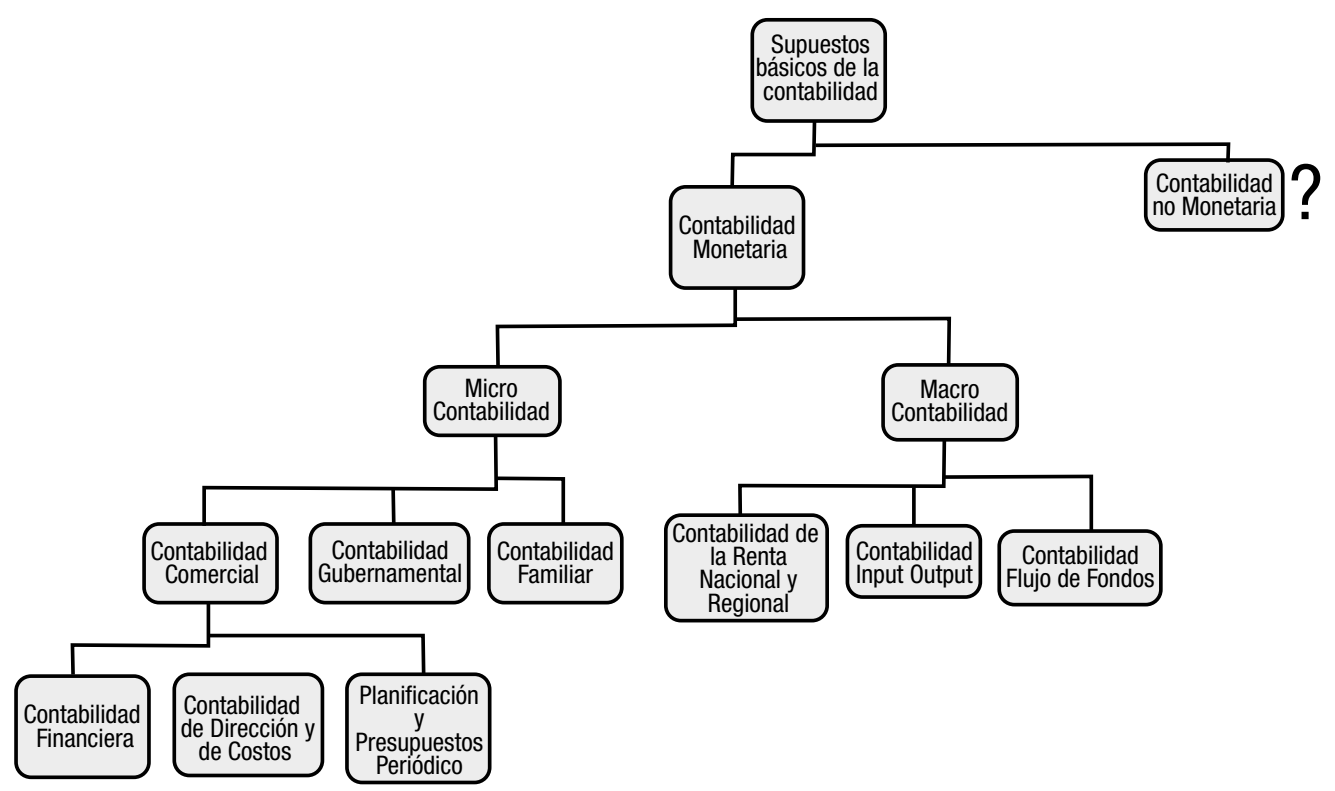

Figura 2. Desarrollo de la contabilidad financiera

Fuente: Elaboración de los autores a partir de Richard Mattessich.

La contabilidad, concebida como un sistema de información que pretende reflejar la situación económica del ente lo más ajustada posible a la realidad, debe mantener en constante revisión y cambio, es decir en revisión continua, máxime cuando los resultados del ejercicio que impactan el patrimonio, son producto de una estrecha relación entre el registro, la medición económica y el reconocimiento y estos atienden a unas metodologías y criterios establecidos en la regulación contable.

Para Díaz (1988), es ciertamente un problema de oportunidad de medición de resultados, el que generará las adecuadas o inadecuadas variaciones de patrimonio. Ateniéndonos a un esquema extremadamente simplista, podemos decir que: "o se miden y registran correcta y oportunamente los resultados, o las participaciones de los propietarios son cifras sin significado real".

\section{La homogeneización de magnitudes en contabilidad}

Para la consecución de la representación de la dimensión económica, existe la necesidad de cambiar las distintas unidades de medida o por lo menos converger a una unidad común 
de medida para las distintas magnitudes; esta unidad de medida tendrá que ser idónea y no hay otra mejor que la monetaria para realizar la convergencia de las magnitudes que se presentan de las categorías que intervienen en las transacciones económicas, lo que origina la existencia del régimen de economía monetaria.

El profesor Requena (1977) distingue en el proceso de medición monetaria tres momentos consecutivos:

1.- Medición física.

2.- Fijación del correspondiente parámetro de conversión, que se conoce como precio de un determinado elemento y representa, por tanto, el valor del mismo.

3.- Expresión monetaria de las unidades físicas obtenidas en la medición a través de la aplicación del coeficiente obtenido en la segunda fase.

\section{Las demandas para el establecimiento de otras dimensiones en la contabilidad}

Se escucharon muchas voces, se leyeron muchos escritos, todos reclamando que a la contabilidad no se le siguiera encasillando únicamente en la revelación de la dimensión económica-financiera; en este documento se presentan algunas de ellas.

Los estudiantes en los pregrados y los profesionales en sus posgrados se enfrentan regularmente a la formulación “¿Existe la contabilidad social?” o “¿Existe alguna relación entre la contabilidad y lo social? Entonces un dejo de incredulidad se sintetiza en esas preguntas, como si se tratara de un discurso coyuntural, retórica circunstancial o planteamiento pseudo científico. Tal designio tiene arraigo en la caracterización de una sociedad que ha puesto en el centro lo económico-financiero y ha dejado en la periferia, al borde del abandono, los aspectos relacionados con la realidad social.

El mundo transita de las visiones segmentadas de carácter cartesiano, a visiones integrales con base en los enfoques holistas y complejos de la filosofía y la ciencia contemporánea. En esa medida, la comprensión de la realidad social requiere de nuevos modelos que incluyan sus diversos atributos y sus relaciones, por tanto, la contabilidad social, más que un discurso o intencionalidad normativa, se erige como una fecunda rama de la contabilidad desde el contexto del conocimiento científico, que brinda respuestas a los problemas sociales, sus causas, manifestaciones y proyecciones en un entorno dinámico. Machado (2004). (Negrilla fuera de texto).

Para Gómez (2006, citado por Mejía 2010 p. 29-30) existen regulaciones contables ambientales donde los elementos desarrollados en dichos modelos no corresponden con las necesidades de información en materia ambiental; es decir; los elementos son inadecuados para producir información relevante útil y pertinente para la toma de decisiones en materia de sostenibilidad socio-ambiental. Esta situación se presenta en virtud de que las preocupaciones ambientales son relativamente recientes -tres décadas- y la articulación contabilidad-medio ambiente obedece a desarrollos emergentes, incluso en construcción. En la actualidad los aspectos financieros siguen primando. 
Según Geba y Dávila (2009)

La especialidad contable socio-ambiental surge entonces, de la creciente responsabilidad social que deben asumir los entes y, con ello la necesidad de elaborar y presentar información sobre las actividades relacionadas con tal responsabilidad. Es decir, los entes deben considerar en la información (contable) los efectos (positivos y negativos) socio-ambientales de su actividad económica

En su trabajo García Casella y Rodríguez de Ramírez (2010) dentro de los avances de su estudio y refiriéndose a las relaciones de la contabilidad con otras disciplinas establecen una serie de conclusiones de las cuales vale la pena destacar que se cuestiona el economicismo de los autores y organismos internacionales que pretenden ubicar a la Contabilidad Social como parte de la Contabilidad Económica, como "cuentas satélites".

Para Barraza y Gómez (2005) la contabilidad ambiental es el campo de conocimiento que estudia sistemas ambientales y naturales, altamente complejos, en su relación con la actividad económica desarrollada por las sociedades, desde luego, su estructura teórica se referencia desde un tipo de racionalidad ambiental, que resulta determinante a la hora de utilizar el hábitat y los recursos naturales. En general, estudia las correspondientes unidades ambientales como totalidades en sus aspectos estáticos como dinámicos, su estructura de relaciones, sus correspondientes impactos y procesos de interacciones bióticas y abióticas.

Para Schmidheiny (1997 citado por Mejía, Montilla y Montes 2010 p.107) los mercados sencillamente no han reflejado con eficiencia los costos de la degradación del medio ambiente. En la mayoría de los casos no se ha conseguido la integración de dichos costos ambientales en las decisiones económicas tanto en el sector empresarial como en el sector gubernamental.

\section{La problemática con las unidades de medida en las dimensiones social y ambiental}

Mucho se ha discutido alrededor de lo que deben ser las unidades de medida para el establecimiento de las dimensiones distintas a la económico-financiera, pues estas definitivamente deben estar alejadas de la unidad monetaria. También son muchos los comentarios que se han escuchado cuando se ha querido representar estas dimensiones social y ambiental en términos monetarios. A continuación se relacionan algunos de los apuntes de distintos autores al respecto.

De acuerdo con Chapman (1981) la problemática de la medición de "los costos y beneficios sociales" es precisamente el de convenir la unidad de medida por utilizar que deberá ser la monetaria por falta de otra más adecuada. Pero esto mismo genera el problema de medir los efectos de la acción [de la organización] sobre valores espirituales...no siempre cuantificables monetariamente.

Conforme a lo expresado por Ablan y Méndez (2004), la interacción de la empresa con el ambiente puede ser medida en términos cuantitativos y cualitativos, financieros y no financieros, no toda la realidad se deja someter al reduccionismo de la valoración monetaria; 
la naturaleza y la sociedad reclaman criterios de valoración socio-ambientales pertinentes para esta realidad. (Negrilla fuera del texto).

En esta misma dirección el profesor Araujo (1995) manifiesta:

(...) ciertamente, medir lo social no puede limitarse a traducir toda la actividad a formas monetarias y por un sistema de cuentas de partida doble. El recurso humano debe mirarse no solo desde su perspectiva económica y financiera, sino también desde una óptica humana, vista como personas que sienten y tienen necesidades que satisfacer. Ello conlleva a producir información que no siempre se puede suministrar en términos monetarios, lo que nos hace pensar en la necesidad de calcular otro tipo de información que sea capaz de traducirnos esa realidad pero expresado en cantidades y cualidades muy diversas; entre otras: los indicadores, los porcientos, las cifras absolutas no monetarias, los índices; así como también formas discursivas que nos hablen de los resultados de una gestión social de la empresa. (Negrilla fuera del texto).

Para Bedford (citado por García Casella, 2001, p.26)

(...) el campo de la Contabilidad debería abarcar cualquier tipo de información necesaria para la toma de decisiones sobre las entidades, es decir, retrospectiva, presente y prospectiva, monetaria y no monetaria; económica y no económica; cualitativa y no cuantitativa; la información debería proporcionarse de acuerdo con las necesidades de los decidores. (Negrilla fuera del texto).

Así mismo para Goldberg (citado por García Casella, 2001, p.25) atar a la contabilidad irrevocablemente a las ocurrencias financieras es demasiado restrictivo y no se compadece con los hechos y los procedimientos contables tal como se llevan a cabo en la actualidad.

Siguiendo a Geba y Dávila (2009) ante la imposibilidad de captar y procesar todas las dimensiones de la realidad es necesario, dentro de un marco teórico, construir modelos que simplifiquen o representen cualidades, rasgos, características, impactos, entre otros, considerados como más relevantes.

\section{La teoría tridimensional de la contabilidad (T3C)}

Ante este cúmulo de reclamaciones, muchos autores han tratado de establecer un marco conceptual para las contabilidades ambiental y social, encontrando hoy un trabajo serio, estructurado, con mucha fundamentación con respecto a lo que se ha pretendido y del cual se espera un constructo y desarrollo sólido que permita superar las taras que hoy tenemos en la contabilidad.

Para Mejía, Montes y Mora (2013) la Teoría Tridimensional de la Contabilidad supera la visión reduccionista de considerar el saber contable solo en la dimensión económicofinanciera. Propone que la contabilidad es una ciencia social aplicada autónoma e independiente, distanciándose de las corrientes que la adscriben como técnica de la economía. La tradicional unidad de medida monetaria, es considerada una posibilidad, pero no la única en el universo de medición/valoración posible de este saber. 
A continuación, se describen, grosso modo, las definiciones planteadas por los autores citados para cada una de las dimensiones contables.

Sociocontabilidad: la contabilidad social es la disciplina social que estudia la valoración cualitativa y cuantitativa de la existencia y circulación de la riqueza social controlada por la organización, utilizando diversos métodos que le permiten cumplir su función de evaluar la gestión que la organización ejerce sobre la riqueza social, con el fin de contribuir a la óptima acumulación, generación, distribución y sostenibilidad integral de la mencionada riqueza [social].

La contabilidad económica como disciplina social que estudia la valoración cualitativa y cuantitativa de la existencia y circulación de la riqueza económica controlada por la organización, utilizando diversos métodos que le permiten cumplir su función de evaluar la gestión que la organización ejerce sobre la riqueza económica, con el fin de contribuir a la óptima acumulación, generación, distribución y sostenibilidad integral de la mencionada riqueza [económica].

La biocontabilidad es la disciplina social que estudia la valoración cualitativa y cuantitativa de la existencia y circulación de la riqueza ambiental controlada por la organización, utilizando diversos métodos que le permiten cumplir su función de evaluar la gestión que la organización ejerce sobre la riqueza ambiental, con el fin de contribuir a la óptima acumulación, generación, distribución y sostenibilidad integral de la mencionada riqueza [ambiental].

La construcción de una estructura general de los modelos contables y el diseño general de los marcos conceptuales para la preparación y presentación de estados e informes contables ambientales, sociales y económicos, pretende cubrir la necesidad de soportar la representación de la realidad contable ambiental, social y económica en parámetros, criterios y conceptos propios de su naturaleza.

Con este trabajo se abren grandes posibilidades para el desarrollo de otras dimensiones de la contabilidad (contabilidad no monetaria) y con el aporte de este trabajo se procura un planteamiento de lo que podrían ser las unidades de medida adoptadas para la representación de las dimensiones ambiental y social a partir de las unidades de medida cualitativas y las unidades de medida cuantitativas no monetarias concebidas dentro de la estructura conceptual de la T3C; además de que se generan serios retos para la profesión contable para el establecimiento de las unidades de medida de algunos fenómenos que se habrán de representar.

\section{Heterogeneización en contabilidad}

$\mathrm{Si}$, definitivamente debemos utilizar otras unidades de medida para las representaciones de las dimensiones social y ambiental dentro de la contabilidad, pues debemos arriesgarnos a proponer esas otras unidades métricas, que no tendremos que homogenizar y que, consideramos, permitirán disminuir el reduccionismo en el que hemos mantenido 
sumergida la contabilidad; en este sentido los futuros estados financieros deberán mostrarse en otras representaciones, otras mediciones. En la Tabla 1 se presentan una serie de variables con sus respectivas unidades de medida, a través de las cuales se podrían realizar las representaciones de las dimensiones citadas.

\section{Tabla 1.}

Magnitudes y unidades de medida cuantitativas no monetarias

\begin{tabular}{|l|l|}
\hline \multicolumn{1}{|c|}{ Variable } & \multicolumn{1}{|c|}{ Unidad de Medida } \\
\hline Demográficas & Unidad hombre \\
\hline Enfermedades profesionales & Tasas de enfermedades prefesionales \\
\hline Morbilidad & Tasas de morbi - mortalidad \\
\hline Ausentismo & Tasas de ausentismo \\
\hline Mortalidad & Tasas de mortalidad \\
\cline { 1 - 2 } Hídricas & $\begin{array}{l}\text { Metros cúbicos, centímetros cúbicos, litros, hectolitros, } \\
\text { megalitros }\end{array}$ \\
\hline Energéticas & $\begin{array}{l}\text { Vatios, kilowatios, vatio/hora, kilovatio/hora, caloría, } \\
\text { termia, frigoria, electronvoltio, caballo de vapor, Ergio, } \\
\text { pie por libra etc. }\end{array}$ \\
\hline Contaminación & Partículas por millón \\
\hline Recurso forestal & Metros cúbicos, centímetros cúbicos, \\
\cline { 1 - 2 } Generación de residuos sólidos & $\begin{array}{l}\text { Metros cuadrados } \\
\text { Metros cúbicos, centímetros cúbicos, litros hectolitros, }\end{array}$ \\
\hline Generación de residuos líquidos & Megalitros \\
\hline Utilización de la tierra & Metros cuadrados \\
\hline Deforestación & Metros cuadrados \\
\hline Pérdida de biodiversidad & Número de especies extintas \\
\hline Exposición a radiación & Gray, Sievert \\
\hline Radiación & Bequerlios, curies, roetgen \\
\hline Adquisición de créditos para emisión de carbono & Metros cúbicos, centímetros cúbicos, \\
\hline Pérdida de fertilidad de tierras & \\
\hline Deterioro de ecosistemas & \\
\hline
\end{tabular}

Fuente: Construcción de los autores.

Partiendo de estas unidades de medida propuestas se presenta en la Tabla 2 lo que puede ser un Estado Contable-socio-ambiental en donde la primera parte de su cuerpo tiene relación con la parte social del componente del talento humano y la segunda parte está relacionada con el desempeño ambiental de una organización económica, se presenta solamente como una aproximación primaria, que mejorada por otros aportes, se espera, sea guía para el desarrollo de la representación contable social y ambiental. 
Tabla 2.

Estado Contable socio-ambiental

Fábrica de muebles Palo Santo Propuesta de estado contable socio-ambiental Año 2015

\begin{tabular}{|l|c|}
\hline \multicolumn{1}{|c|}{ CONCEPTO } & CANTIDAD \\
\hline \multicolumn{1}{|c|}{ Desempeño social-Talento Humano } & 72 \\
\hline Cantidad de empleados & 59 \\
\hline Cantidad de empleados sindicalizados & 0,2 \\
\hline Morbilidad & 0,01 \\
\hline Mortalidad & 0,25 \\
\hline Ausentismo & 2 \\
\hline Pensionados & 5 \\
\hline Cursos de formación & \\
\hline \multicolumn{1}{|c|}{ Desempeño ambiental } & 10 metros cúbicos \\
\hline Consumo de agua potable & 256,4 metros cúbicos \\
\hline Consumo de agua no potable & 3,200 kilómetros cúbicos \\
\hline Contaminación de agua & 4.532 kilowatios/hora \\
\hline Consumo de energía eléctrica & 547 kilos \\
\hline Consumo de carbón & 1694 metros cúbicos \\
\hline Consumo de gas natural & 458 Metros cúbicos \\
\hline Consumo de recurso forestal (madera) & 984 kilos \\
\hline Generación de residuos sólidos & 93 litros \\
\hline Generación de residuos líquidos & 125 metros cúbicos \\
\hline Emisión de contaminantes & 56 kilos \\
\hline Cantidad de material reutilizable & \\
\hline
\end{tabular}

Notas al Estado Contable socio-ambiental:

La entidad tiene dentro de su plan de salud los siguientes programas:

1. Para prevenir lesiones osteomusculares.

2. Vigilancia epidemiológica para conservación respiratoria.

Se realizaron los siguientes cursos de formación:

1. Riesgos profesionales en la industria maderera

2. El cuidado de las vías respiratorias

3. Prevención de riesgos eléctricos

4. Las micro finanzas en la familia

5. El cuidado de la salud, posturas y flexiones

Existe una convención colectiva de trabajo

Fuente: Construcción de los autores 


\section{Conclusiones}

La medición y la valoración en contabilidad han estado estrechamente ligadas, lo que hace que se fundan y también que se confundan, esto pasa generalmente con aquellos profesionales que no han ahondado en el estudio de la contabilidad, en su estructura y composición y permanentemente establecen la relación medición-contabilidad.

Con propuestas como la T3C, planteada por los profesores Mejía, Montes y Mora se abre un abanico de posibilidades para el desarrollo de la contabilidad desde la óptica no financiera y por ende se puede generar crecimiento para la contaduría pública, se generan más desafíos para la profesión contable que debe realizar aportes ya no desde la reduccionista visión económico-financiera, sino desde otras perspectivas.

La contabilidad es una ciencia social aplicada y como tal no se deben descuidar los aspectos atinentes al medioambiente, a lo específicamente social, por ende, tenemos que seguir en la búsqueda del posicionamiento de las dimensiones social y ambiental.

De la misma manera, con el establecimiento de una estructura que configura otras dimensiones de la contabilidad se está contrariando a algunos autores clásicos como el profesor Fernández Pirla (1970) quien manifestó que

Toda formulación contable descansa en el postulado fundamental de permanencia en el patrón monetario de medida". Del mismo modo afirma que "cuando la estabilidad monetaria queda alterada, la Contabilidad pierde su significado, su función representativa, y pasa a exponer un conjunto heterogéneo de valores no comparables entre sí, porque vienen expresados en distintas unidades monetarias de medida que están dadas por los diversos poderes de adquisición del dinero correspondiente a las fechas en que fueron realizadas las inversiones.

\section{Conflicto de intereses}

Los autores declaran no tener ningún conflicto de intereses.

\section{Referencias bibliográficas}

1. Ablan Bortone, N. y Méndez Vergara, E. (2004). Contabilidad y ambiente: Una disciplina y un campo para el conocimiento y la acción. Revista Actualidad Contable FACES 7 (8). 7-22.

2. Andriessen D. (2004).”Intellectual capital valuation and measurement: classifiying the state of the art”. Journal of Intellectual capital. 5 (2) 230-242

3. Araujo Ensuncho J. A. (1995). Contabilidad social. Centro Colombiano de investigación Contable. C-CINCO. Medellín.

4. Barraza Caro, Frank Eduardo y Gómez Santrich, Martha Liliana, (2005). Aproximación al concepto de contabilidad ambiental. Bogotá: Universidad Cooperativa de Colombia.

5. Chapman, W. L. (1981) Dificultades para medir la cuantía del beneficio social neto de la actividad económica de las empresas públicas y privadas. Anales de la Academia Nacional de las Ciencias Económicas, vol. XXVI. Buenos Aires, Argentina. 
6. Díaz Castro, J. (1988). Análisis crítico de la información contable. Revista de investigación contable Teuken (2).

7. Fernández, L. y Bifaretti, M. (2010) Marco conceptual para la especialidad contable socio-ambiental. Revista de la universidad de los Andes Venezuela. (20)

8. García Casella, C. L. (2001). Elementos para una teoría general de la contabilidad. Buenos Aires. La Ley.

9. Habdelgani H. N. y Lemos de la Cruz J. E. (2010). Reflexión sobre el obstáculo epistemológico del concepto de medición en contabilidad. Revista Libre Empresa 7(2) 101-115

10. Geba, N. B. y Dávila, G. C, (2009). Epistemología en contabilidad, modelos contables. En 15to Encuentro nacional de investigadores universitarios del área contable.

11. Ijiri, Yuji, (1967). The Foundations of Accounting Measurement, Prentice Hall Inc. Englewood Cliffs.

12. Mattessich, Richard. Contabilidad y métodos analíticos. Buenos Aires: La Ley. 2002.

13. Mejía Soto, Eutimio, (2010). Contabilidad ambiental, crítica al modelo de contabilidad financiera. Armenia.

14. Mejía, E., Montes, C. A. (2012). Análisis de los conceptos de capital, mantenimiento de este y su relación con la riqueza ambiental. En revista internacional Legis de contabilidad y auditoría $\mathrm{N}^{\circ} 49$ enero-marzo.

15. Mejía, E., Montes, C. A. y Botero Echeverry, D. (2006). Programas de investigación en contabilidad. Cali: Artes Gráficas.

16. Mejía, E., Montes, C. A. y Mora, G. (2013). Estructura conceptual de la Teoría Tridimensional de la Contabilidad. Revista Contexto (2), 34-53.

17. Mejía, E., Mora, G. y Montes, C. A. (2013). Adscripción de la contabilidad en la estructura general del conocimiento. Revista Cuadernos de Contabilidad (34) 159-187.

18. Mejía, E, Montilla, O. de J. y Montes, C. A. (2010). Análisis de los métodos de medición de las cuentas ambientales en el modelo contable financiero y concepciones alternativas. Entramado 6 (2) 106-128.

19. Perea Román F. (1988). Hacia un nuevo concepto: Sociocontaduría. Revista de investigación contable Teuken (2).

20. Requena Rodríguez, J. M. (1977). La homogeneización de magnitudes en la ciencia de la Contabilidad, I.C.E., Madrid. 